\title{
Medicina Integrativa: um parecer situacional a partir da percepção de médicos no Sul do Brasil
}

\author{
Integrative Medicine: situational assessment through medical \\ perception in the South of Brazil
}

Maria Luiza Amaral Kracik', Pablo Michel Barcelos Pereira', Betine Pinto Moehlecke Iser $\mathbf{1}$

DOI: 10.1590/0103-1104201912309

RESUMO O estudo teve por objetivo identificar o interesse, a concordância e o conhecimento dos médicos atuantes no Sistema Único de Saúde (SUS) no município de Tubarão (SC) em relação às práticas da Medicina Integrativa e sua política nacional de inclusão ao sistema de saúde. Foi realizado estudo transversal, em amostra do tipo censo, composta por um total de 230 profissionais. A maioria dos médicos investigados referiu desconhecer a Política Nacional de Práticas Integrativas e Complementares (85,5\%) e negou possuir interesse pelas suas modalidades terapêuticas (53,3\%). Ainda assim, um número significativo deles referiu querer conhecer mais sobre as práticas (43\%) e concordar com a inclusão delas no SUS (80,2\%), sendo que $48,8 \%$ concordam somente com a inclusão de algumas práticas. O uso próprio ou de familiares influenciou no conhecimento e, com isso, na recomendação de uso aos pacientes.

PALAVRAS-CHAVE Medicina Integrativa. Médicos. Sistema Único de Saúde.

\begin{abstract}
The study aimed to identify the interest, agreement and knowledge of the doctors working in the Unified Health System (SUS) in the city of Tubarão (SC) regarding the practices of Integrative Medicine and their national policy of inclusion in the health system. A cross-sectional study was conducted on a census-type sample composed of a total of 230 professionals. Most investigated doctors reported not knowing the national policy of integrative and complementary practices (85.5\%) and denied having interest in their therapeutic modalities (53.3\%). Even so, a significant number of them reported wanting to know more about the practices (43\%) and agreeing to include them in the SUS (80.2\%), and $48.8 \%$ agree only with the inclusion of some practices. The use of one's own or family members influenced the knowledge and, therefore, the recommendation of use to the patients.
\end{abstract}

KEYWORDS Integrative medicine. Doctors. Unified Health System.

1 Universidade do Sul de Santa Catarina (Unisul), Programa de Pós- 


\section{Introdução}

Com o advento da crise na economia e na saúde pública, desde o início do século XX, a Organização Mundial de Saúde (OMS) optou por utilizar os recursos da medicina tradicional e da cultura popular' ${ }^{1}$. Essa foi uma das propostas lançadas na Conferência de Alma-Ata para confrontar a falta de cobertura básica de saúde, pois tais práticas já eram utilizadas em diferentes culturas respeitando suas peculiaridades ${ }^{\mathbf{2}, 3}$.

Nos últimos anos, houve um incremento da procura por formas de cuidado com a saúde diferente dos padrões convencionais, buscando uma abordagem de cuidar do sujeito de forma integral (corpo, mente e espírito) ${ }^{3}$. Em consequência, o crescimento vertical da sua oferta e uso tem estimulado discussões acadêmicas em torno da utilização segura e eficaz dessas modalidades terapêuticas ${ }^{4}$.

A Medicina Integrativa (MI), segundo alguns autores ${ }^{5,6}$ veio para preencher a lacuna da saúde tradicional, podendo ser descrita como o conjunto de práticas em saúde que combina técnicas não alopáticas à medicina moderna, a qual se sustenta em quatro pilares:1) tratamento do indivíduo como um todo, e não de maneira cartesiana; 2) relação não hierárquica e interdisciplinar com a Medicina Alopática Convencional (MAC); 3) abordagem multidisciplinar e construção de consensos; 4) cuidado com efetividade e custo acessível ${ }^{3-7}$.

Identifica-se dentro da MI uma diversidade de práticas, algumas delas são: medicina antroposófica, termalismo, naturopatia, aromoterapia, fitoterapia, shiatsu, Lian gong, chás, reflexologia, osteopatia, massoterapia, ioga, meditação, oração, xantala, quiropraxia, ayuverda, tai chi, Reik, entre outras, como a homeopatia e a acupuntura, que são regulamentadas como especialidades médicas no Brasil2-10.

No século XX, um terço da população dos Estados Unidos referia usufruir de, pelo menos, uma dessas modalidades terapêuticas supracitadas, e estima-se que atualmente essa razão tenha aumentado consideravelmente ${ }^{11-14}$. De fato, nos últimos 25 anos, o seu uso aumentou em países ocidentais, principalmenteentre os industrializados ${ }^{6,11}$.
O reconhecimento dessas práticas no Brasil, com o aumento da demanda por esses serviços, motivou a implementação, em 2006, da Política Nacional de Práticas Integrativas e Complementares (PNPIC), que normatizou e regularizou a prática da MI no País 5 .

Devido à descentralização do Sistema Único de Saúde (SUS), e consequente autonomia estadual e municipal do sistema, as Práticas Integrativas e Complementares (PIC) já eram ofertadas antes mesmo da inserção oficial da PNPIC no País, pois, desde a década de 1970, a OMS incentivava sua prática mundialmente ${ }^{3-15}$. Segundo o Ministério da Saúde (MS):

[...] em 2017, 8.200 Unidades Básicas de Saúde (UBS) ofertaram alguma das PICS, o que corresponde a $19 \%$ desses estabelecimentos. Essa oferta está distribuída em 3.018 municípios, ou seja, $54 \%$ do total, estando presente em $100 \%$ das capitais por iniciativa das gestões locais. Em 2016, foram registrados oferta em PICS em 2.203.661 atendimentos individuais e 224.258 atividades coletivas, envolvendo mais de 5 milhões de pessoas ${ }^{5(10)}$.

Entretanto, a dificuldade da sua conceituação e normatização fornecem diferentes significâncias e interpretações para essa modalidade de medicina, até mesmo diferentemente nomeada, como complementar, alternativa, integrativa, popular ${ }^{2-9}$, fazendo com que a consistência das pesquisas nessa área permaneça diminuída ${ }^{15-18}$ e reste poucas informações sobre a segurança e a eficácia de suas práticas ${ }^{4-7}$.

Essas lacunas de conhecimento sobre as PIC comprometem seu papel e uso apropriados dentro da área da saúde ${ }^{3-5}$, sendo encontradas barreiras à aceitação de tais práticas por parte da medicina alopática, regida pela filosofia da medicina baseada em evidências ${ }^{7}$. De fato, o uso das PIC em saúde parece ser subestimado por médicos, que dificilmente incluem, durante uma consulta, perguntas sobre a utilização de alguma prática não convencional ${ }^{\mathbf{1 3}}$. Apesar dessas questões, tem-se percebido 
que essas práticas suscitam cada vez mais o interesse popular6,9,14,15. Com o alto custo da biomedicina, o elevado potencial da MI como promotora de saúde e geradora de bem-estar, e com o aumento das doenças crônicas ${ }^{12}$, da expectativa de vida e da procura por uma maior qualidade de vida, a MI intensifica o senso e capacidade de autocuidado ${ }^{1}$, sendo aliada fundamental na manutenção da saúde10,11,15.

Considerando que a MI está ganhando espaço entre a política de saúde no Brasil e, devido ao aumento da demanda por PIC, faz-se necessário que o profissional médico se molde a essa demanda de maneira a poder orientar seus pacientes com segurança a respeito da indicação ou contraindicação de qualquer modalidade integrativa. Dessa forma, o presente estudo objetivou identificar o interesse, a concordância e o conhecimento dos médicos atuantes no SUS do município de Tubarão, em Santa Catarina (SC), em relação às práticas da MI e sua política nacional de inclusão ao sistema de saúde.

\section{Métodos}

Realizou-se estudo observacional de delineamento transversal. Efetuou-se amostragem do tipo censo, a partir de uma população composta por 230 médicos atuantes no município, inferida por meio do acesso autorizado pela secretaria de saúde do município às listas que contêm o corpo clínico das Unidades Básicas de Saúde (UBS) e do Hospital Nossa Senhora da Conceição, vinculado à universidade.

Como critério de inclusão, estabeleceu-se que os profissionais deveriam atuar no município de Tubarão e ter, obrigatoriamente, vínculos empregatícios com o SUS. Excluíram-se os médicos cuja atividade clínica não incluísse indicação terapêutica direta aos pacientes -por exemplo, radiologistas -, bem como aqueles que não foram encontrados nos locais de trabalho no período de coleta de dados em função de afastamento, férias ou licença, ou que não aceitaram participar do estudo mediante assinatura do Termo de Consentimento Livre e Esclarecido (TCLE).
Os dados necessários à pesquisa foram extraídos mediante o uso de um questionário baseado na pesquisa desenvolvida por Faqueti e colaboradores ${ }^{12-19}$. $\mathrm{O}$ instrumento foi criado e testado, pelos autores do referido projeto, e disponibilizado aos pesquisadores mediante contato prévio.

O questionário foi composto por nove questões sociodemográficas e de atuação profissional e por outras dez referentes à percepção, ao conhecimento, ao uso e interesse dos profissionais sobre a MI. A primeira questão do questionário específico buscou inferir sobre como o entrevistado percebia as PIC. Três questões buscaram apontar o conhecimento, a aceitação e a concordância com a PNPIC. Quatro questões inferiram sobre o interesse e o conhecimento sobre a oferta de PIC no município, além de uma pergunta com o intuito de identificar se o médico reconhecia o interesse dos seus pacientes/comunidade por essas práticas. $\mathrm{O}$ conhecimento propriamente dito sobre essas práticas foi avaliado na última questão, a qual permitiu conhecer o padrão de uso e posicionamento, em se tratando de recomendação, sobre as PIC. O instrumento foi entregue pessoalmente ao profissional médico para autopreenchimento, podendo ele escolher o local e momento de preenchê-lo.

As variáveis independentes foram as características individuais e profissionais, e as variáveis desfecho foram o conhecimento, o interesse e a concordância/aceitação em relação às PIC e sua política nacional. $\mathrm{O}$ interesse foi considerado como 'sim', para aqueles que marcaram que gostariam de conhecer mais, gostariam de especializar-se ou realizar capacitação na área, e 'não' quando não tem nenhum interesse ou tem pouca curiosidade.

Da mesma maneira, a concordância foi considerada como 'sim' para aqueles que afirmaram que concordam plenamente ou com a inclusão de algumas práticas. $\mathrm{O}$ conhecimento, uso e recomendação/encaminhamento foi entendido como 'sim' para as respostas: eventualmente/ alguma coisa, bastante/muito e sempre/é para cada prática investigada. Para as práticas mais populares, investigou-se também a relação entre o conhecimento e o uso pessoal ou familiar com a 
recomendação ou prescrição dela aos pacientes.

Os dados foram tabulados e analisados por meio do programa EpiInfo 3.5.4. A apresentação dos dados foi realizada por meio da estatística descritiva, sendo as variáveis qualitativas expressas em proporções; e as variáveis quantitativas, em medidas de tendência central e dispersão adequadas, de acordo com a normalidade dos dados. Para verificar a associação entre as variáveis de interesse, utilizaram-se os testes Qui-quadrado de Pearson e t de Student para a comparação entre médias, ou correspondentes não paramétricos. $O$ nível de significância preestabelecido foi de $5 \%$.

O projeto foi aprovado pelo Comitê de Ética em Pesquisa da Universidade do Sul de Santa Catarina (Unisul), sob parecer $\mathrm{n}^{\circ} 770.828$. Em respeito à Resolução ${ }^{0} 466$ de 2012 do Conselho Nacional de Saúde, foram oferecidos a todos os participantes o TCLE, sendo-lhes garantido anonimato, sigilo de informações, explanações a respeito do estudo, bem como autonomia para decidir sobre sua participação.

\section{Resultados}

O estudo contou com a participação de 172 profissionais médicos, $74,7 \%$ da população pretendida inicialmente. Um respondente não preencheu adequadamente o último bloco do questionário, o qual inquere sobre o conhecimento e sobre uso pessoal das práticas integrativas, sendo excluído desta análise. Houve maior representação do sexo masculino (57,6\%), com idade média de 38,9 anos $( \pm 10,7)$, casados ou em união estável (65,2\%) e com, pelo menos, um filho (58,7\%).

Em geral, os profissionais têm residência médica ( $75 \%)$ ou estão cursando uma (9,3\%). Dentre elas, destacam-se: clínica médica (16,9\%); ginecologia/obstetrícia (7\%); pediatria (7\%); cirurgia geral $(5,8 \%)$ e saúde da família (4,7\%). A maioria é formada há mais de 11 anos (55\%), atua no Hospital Nossa Senhora da Conceição (87,2\%), e exerce a profissão simultaneamente pelo serviço público e privado de saúde (84,3\%). Apenas 19 médicos (11\%) referiram trabalhar exclusivamente em UBS.

Predominaram entre os médicos aqueles que nunca cursaram qualquer disciplina relacionada com as práticas integrativas (75\%), sendo percebidas pela maioria como complementares (70,3\%). Uma parcela dos investigados acredita que elas estejam relacionadas com o efeito placebo (14\%) e/ou não tenham validade científica (10,5\%), devendo ser utilizadas somente se comprovadas cientificamente (47,1\%) (tabela 1).

Tabela 1. Percepção geral dos médicos investigados sobre as diversas Práticas Integrativas e Complementares

\begin{tabular}{lcr}
\hline Variáveis & $\mathbf{N}=\mathbf{1 7 2}$ \\
\hline Percepção' $^{17}$ & $\mathbf{n}$ \\
São práticas complementares & 70,3 & 121 \\
Só usar as comprovadas cientificamente & 47,1 & 39 \\
Representam diferente entendimento do processo saúde-doença & 22,7 & 34 \\
Representam amplo entendimento do processo saúde-doença & 19,8 & 24 \\
Relacionadas com o efeito placebo & 14,0 & 18 \\
Não têm validade científica & 10,5 & 18 \\
São independentes da biomedicina & 10,5 & 6 \\
Não têm opinião a respeito & 3,5 & 39 \\
\hline
\end{tabular}

Fonte: Elaboração própria.

1 Permite mais de uma resposta. 
A maioria dos médicos investigados referiu desconhecer a PNPIC $(85,5 \%)$ e negou possuir interesse pelas suas modalidades terapêuticas (53,3\%), sendo pequena a parcela dos que desejam se especializar ou se capacitar nessa área médica $(3,5 \%)$. Ainda assim, um número significativo deles referiu querer conhecer mais sobre as práticas (43\%) e concordar com a inclusão delas no SUS (80,2\%), sendo que $48,8 \%$ concordam somente com a inclusão de algumas práticas.
O reconhecimento da necessidade da abordagem sobre MI no meio acadêmico como disciplina optativa ou obrigatória foi alto (83,7\%); e, excetuando-se aqueles que acreditam que a inclusão de ofertas não deva ocorrer em nenhum nível ou exclusivamente na atenção especializada (secundária), 70,4\% referiram ser a favor da oferta delas em pelo menos um nível: a atenção básica (tabela 2).

Tabela 2. Representação do posicionamento dos médicos ante as Práticas Integrativas e Complementares e a sua política nacional de inserção no SUS

\begin{tabular}{|c|c|c|}
\hline Variável & $\%$ & $\mathrm{n}$ \\
\hline \multicolumn{3}{|l|}{ Conhecimento sobre PNPIC ${ }^{\star}$} \\
\hline Sim & 14,5 & 25 \\
\hline Não/Pouco & 85,5 & 147 \\
\hline \multicolumn{3}{|c|}{ Concordância com a inclusão no SUS } \\
\hline $\operatorname{Sim}$ & 80,2 & 138 \\
\hline Não & 19,8 & 34 \\
\hline \multicolumn{3}{|l|}{ Nível de inserção no SUS } \\
\hline Em todos os níveis & 28,5 & 49 \\
\hline Atenção primária e secundária & 10,5 & 18 \\
\hline Atenção primária & 31,4 & 54 \\
\hline Atenção secundária & 14,5 & 25 \\
\hline Não sabe/Nenhum & 15,1 & 26 \\
\hline \multicolumn{3}{|l|}{ Interesse por $\mathrm{PIC}_{\dagger}$} \\
\hline $\operatorname{Sim}$ & 46,5 & 80 \\
\hline Não/Pouco & 53,3 & 92 \\
\hline \multicolumn{3}{|l|}{ Inclusão na graduação } \\
\hline Optativa & 51,7 & 89 \\
\hline Obrigatória & 32,0 & 55 \\
\hline Extensão ou Especialização & 10,5 & 18 \\
\hline Não é necessária & 5,8 & 10 \\
\hline TOTAL & 100 & 172 \\
\hline
\end{tabular}

Fonte: Elaboração própria.

*PNPIC: Política Nacional de Práticas Integrativas e Complementares.

†PIC: Práticas Integrativas e Complementares. 
Grande parte dos médicos investigados não percebe o interesse dos seus pacientes/comunidade pelas práticas integrativas (76,7\%), e a oferta delas pelo SUS no município é desconhecida por $56,4 \%$. Outros $68,8 \%$ afirmaram não ter essa oferta em seu local de trabalho (tabela 3). A acupuntura (58,6\%), a homeopatia (12,6\%), o Reiki (6,9\%) e a fitoterapia $(4,6 \%)$ foram as práticas mais citadas entre aqueles que referiram a disponibilidade de ofertas pelo serviço público no município (gráfico 1).

Tabela 3. Conhecimento dos médicos investigados sobre a disponibilidade de ofertas de Práticas Integrativas e Complementares e do interesse comunitário por elas

\begin{tabular}{lrr}
\hline Variável & $\%$ & $\mathbf{n}$ \\
\hline Existência de oferta no município & & 65 \\
\hline Sim & 37,8 & 10 \\
Não & 5,8 & 97 \\
Não sabe & 56,4 & 31 \\
\hline Existência de oferta no local de trabalho & 18,0 & 118 \\
\hline Sim & 68,8 & 23 \\
Não & 13,4 & 40 \\
Não sabe & & 132 \\
\hline Interesse da comunidade & 23,3 & $\mathbf{1 7 2}$ \\
\hline Sim & 76,7 & 100 \\
Não/não sabe & 100 \\
\hline Total & & \\
\hline
\end{tabular}

Fonte: Elaboração própria.

Gráfico 1. Oferta de práticas existentes no município de Tubarão (SC) e nos locais de trabalho segundo os médicos atuantes no município

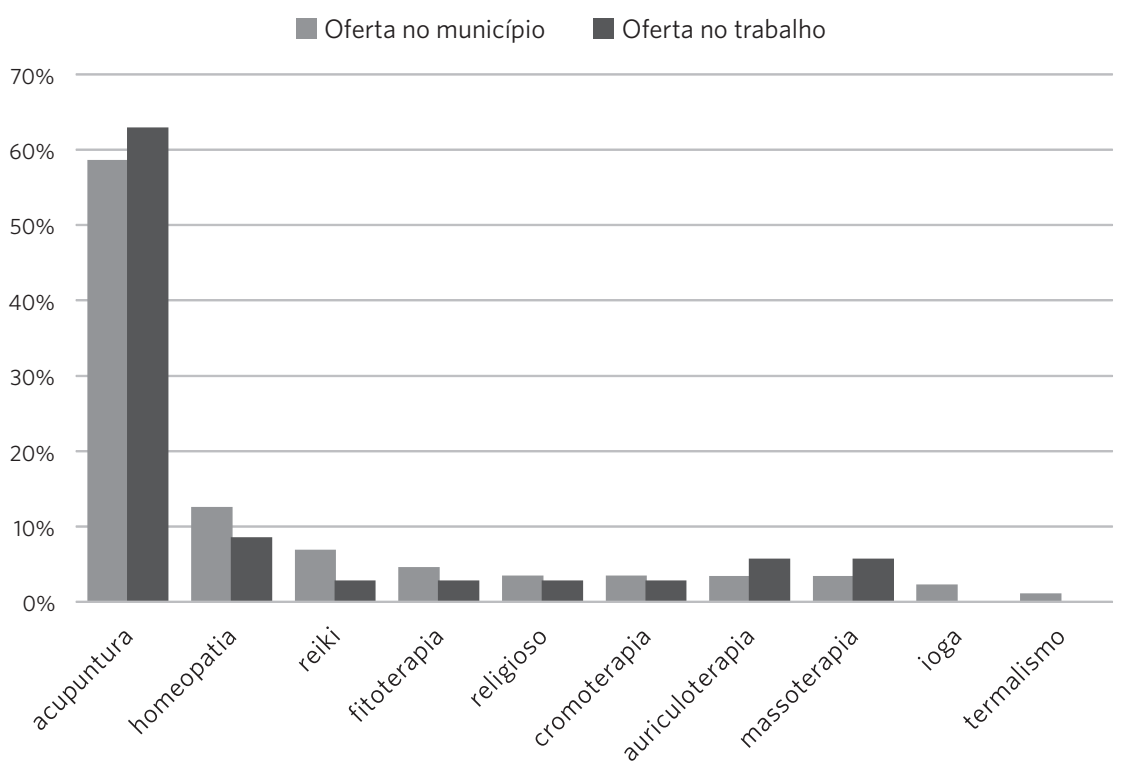


De fato, a acupuntura foi a modalidade com maior nível de conhecimento $(61,4 \%)$ e recomendação (43,8\%), estando à frente de todas as demais práticas investigadas (tabela 4). As práticas mais utilizadas pelos profissionais médicos ou seus familiares foram as de cunho espiritual/religioso $(65,1 \%)$ e massagem $(62,5 \%)$. As mais prescritas foram a fitoterapia $(18,7 \%)$ e a acupuntura (16,3\%).

Tabela 4. Conhecimento, uso (pessoal e/ou familiar), recomendação e prescrição das PIC referidos pelos profissionais médicos atuantes no SUS, de acordo com cada prática integrativa e complementar. Tubarão, SC, 2014

\begin{tabular}{lrrrrrrrr}
\hline Práticas & & Conhece & & Usa & \multicolumn{2}{c}{ Recomenda } & \multicolumn{2}{r}{ Prescreve } \\
\cline { 2 - 8 } & $\mathbf{n}$ & $\mathbf{9}$ & $\mathbf{n}$ & $\%$ & $\mathbf{n}$ & $\%$ & $\mathbf{n}$ & $\%$ \\
\hline Homeopatia & 52 & 30,4 & 15 & 8,8 & 10 & 5,9 & 1 & 0,6 \\
Acupuntura & 105 & 61,4 & 62 & 36,3 & 75 & 43,8 & 28 & 16,3 \\
Medicina Chinesa & 6 & 3,5 & - & - & - & - & - & - \\
Medicina Ayurveda & 1 & 0,6 & - & - & - & - & - & - \\
Medicina Antroposófica & - & - & - & - & - & - & - & - \\
Fitoterapia & 49 & 28,6 & 43 & 25,2 & 37 & 21,6 & 32 & 18,7 \\
Ervas Medicinais & 16 & 19,4 & 10 & 5,8 & 7 & 4,1 & 1 & 0,6 \\
Práticas corporais & 52 & 30,4 & 32 & 18,8 & 29 & 17,0 & 7 & 4,1 \\
Massagem & 69 & 40,3 & 107 & 62,5 & 53 & 31,0 & 11 & 6,4 \\
Reiki & 18 & 10,6 & 14 & 8,2 & 3 & 1,8 & - & - \\
Toque terapêutico & 1 & 0,6 & - & - & - & - & - & - \\
Espiritual/ Religioso & 74 & 43,2 & 111 & 65,1 & 33 & 19,3 & 4 & 2,4 \\
\hline Total & $\mathbf{1 7 1}$ & $\mathbf{1 0 0}$ & $\mathbf{1 7 1}$ & $\mathbf{1 0 0}$ & $\mathbf{1 7 1}$ & $\mathbf{1 0 0}$ & $\mathbf{1 7 1}$ & $\mathbf{1 0 0}$ \\
\hline
\end{tabular}

Fonte: Elaboração própria.

O uso de alguma prática, seja para si ou para familiares, teve relação significativa com $o$ ato de recomendá-la ou prescrevê-la. O uso da homeopatia influiu positivamente para a sua recomendação ( $\mathrm{p}=0,00008)$, e o mesmo aconteceu para a acupuntura $(\mathrm{p}=0,00001)$, para a fitoterapia $(\mathrm{p}=0,00001)$, para as práticas corporais $(\mathrm{p}=0,00001)$ e para a massagem $(\mathrm{p}=0,00001)$.

O conhecimento sobre determinadas práticas também aumentou significativamente a frequência de recomendação delas: a homeopatia $(p=0,001)$, a acupuntura $(p=0,00001)$, a fitoterapia ( $\mathrm{p}=0,00001)$, as práticas corporais $(p=0,00001)$ e a massagem $(p=0,00001)$.

A abordagem ou a capacitação realizadas na graduação ou após sua formação básica não tiveram relação com o nível de conhecimento que o profissional referiu para cada prática, tampouco com o ato de recomendar alguma modalidade, à exceção da homeopatia, única racionalidade que teve a recomendação $(\mathrm{p}=0,02)$ e o conhecimento $(\mathrm{p}=0,05)$ influenciados pela abordagem acadêmica.

As características pessoais - sexo, idade, estado civil, número de filhos - e profissionais - ano de formado, tempo de atuação no SUS, especialidade clínica ou cirúrgica - não apresentaram influência em relação ao conhecimento, ao uso, à recomendação e à prescrição das práticas integrativas. 


\section{Discussão}

O presente estudou contou com uma amostra diferenciada dos demais por ser composta majoritariamente por médicos atuantes em ambiente intra-hospitalar na qualidade de especialistas ou residentes. Islândia et al. ${ }^{\mathbf{2 0}}$, ao analisar a oferta e a produção de atendimentos no SUS em três diferentes cidades, constatou maior concentração de práticas integrativas na atenção básica de saúde. Outros autores ${ }^{2,4,9}$ apontam as UBS como porta de entrada para as PIC, e sinalizam uma associação entre a medicina da família com uma melhor percepção e afinidade pelas práticas.

Presume-se, portanto, que médicos da atenção primária nutram não apenas maior apreço pelas práticas como também tenham mais conhecimento sobre elas, provavelmente por ser um conteúdo oferecido durante a especialização na área, ainda que de forma optativa ${ }^{21}$. Contudo, no que diz respeito ao conhecimento sobre o universo da MI, evidenciou-se aqui condição contrária ao evidenciado na literatura e, portanto, ao esperado no estudo.

O desconhecimento dos médicos especialistas (que atuam majoritariamente em ambiente intra-hospitalar) mostrou-se similar ao dos médicos centrados na atenção primária (vinculados às UBS) avaliados em outras pesquisas $\mathbf{2}^{\mathbf{2}, \mathbf{2 0}}$. Portanto, a compreensão para o desconhecimento da PNPIC e suas práticas pode estar situada em outros horizontes que não na diferença entre os níveis de atenção em saúde.

Em se tratando de avaliar o crescimento da MI e suas práticas no Brasil, deve-se ter em conta sua heterogeneidade e descentralização do sistema de saúde. Avaliando-se por meio do Cadastro Nacional de Estabelecimentos de Saúde (CNES), nota-se diferenças na distribuição de ofertas entre cidades, o que caracteriza pouca homogeneidade na disponibilidade delas. Neste estudo, muitos médicos desconheciam a oferta de terapias complementares no município e até mesmo em seu local de trabalho.
De fato, é amplamente evidenciada e difundida a existência de diferenças na aceitação e padrão de uso de PIC entre cidades de uma mesma Unidade da Federação. Assumese que diferenças culturais e de disponibilidade de ofertas sejam um diferencial na familiarização da comunidade médica e não médica com a MI9,20,22.

Em estudo semelhante a este, em Florianópolis, encontraram-se profissionais com percepções mais positivas e interesses maiores dos que os demonstrados pelos médicos investigados neste estudo. Pode-se justificar esse achado pela diferença da amostra utilizada na capital de SC, centrada na atenção primária, e pelo maior número de médicos que relataram ser especialistas em medicina da família, e/ou capacitados em homeopatia ou acupuntura ${ }^{17,20}$.

Ainda que a maioria dos profissionais tenha referido desinteresse pelas PIC, existe uma parcela importante que refere querer conhecer mais sobre elas. A opinião dividida sobre as PIC é mais comumente encontrada entre os médicos se comparada às demais categorias profissionais dentro da área da saúde $\mathbf{1 8 , 2 2}^{\mathbf{2 2}}$

Boa parte da divergência de opiniões reflete o impasse entre o MS, o Conselho Federal de Medicina (CFM) e o Ministério de Educação e Cultura (MEC). A aplicação fitoterápica no tratamento de doenças ainda não é reconhecida pelo CFM, no entanto, existe o estímulo ao seu uso por meio da implantação de cursos de capacitação em fitoterapia pelo MEC, corroborando a inclusão dessa prática no SUS por intermédio da Portaria GM no 971 do $\mathrm{MS}^{23}$.

Outro fator importante que fomenta a divergência de opinião sobre a MI recai sobre a formação acadêmica durante a graduação, com foco quase que puramente alopático. Somando-se a isso, a maior dependência no arsenal tecnológico por ela oferecido também contribui para a visão rígida e objetiva que divide o homem em partes, afastando-o da visão mais humana/ emocional, essencial para a construção da relação médico-paciente. 
No que tange ao estudo da ética médica, o avanço da biotecnologia dentro da medicina fez com que o homem se tornasse objeto da técnica criada e aperfeiçoada por ele próprio. Alguns autores ${ }^{2-8}$ descrevem a relação médico-paciente como algo superior à habilidade profissional adquirida pelo médico, dizendo ser o somatório de empatia, conjunto de destreza pessoal e técnicas.

A visão e o autocuidado corporal, o senso de preservação, os valores pessoais, sociais e familiares, as crenças e as atitudes diante da vida e da morte são partes consideradas essenciais para a MI, sendo necessário ao médico ter uma visão mais complexa que ultrapasse o diagnóstico da doença para tratar o indivíduo integralmente.

Existem evidências que demonstram que pessoas que buscam terapêuticas dentro da MI buscaram primeiro auxílio na medicina alopática, não obtendo resposta satisfatória mesmo com o seu amplo arsenal de terapêuticas ${ }^{10,12,21}$.

A baixa percepção que o médico tem sobre o real interesse da comunidade pelas PIC é corroborada pelo que é descrito em literatura. Diversas situações sustentam a hipótese de que não haverá qualquer comunicação a respeito de alguma prática se o paciente não questionar sobre a adoção delas9,11.

A comunidade não se sente confortável em informar ao seu médico sobre o uso de alguma modalidade, tampouco fazer questionamentos a respeito dela, por acreditar que terá sua iniciativa criticada. Em contradição, muitas vezes o médico se queixa sobre o fato de não concordar com a adoção de PIC por serem indicadas por outros profissionais da área da saúde ${ }^{12,16,20}$.

Atualmente, devido à maior demanda que tem se apresentado pelas PIC, a aceitação dos médicos nos diversos estados brasileiros tem também aumentado, principalmente quando são práticas outorgadas pelo CFM. A acupuntura e homeopatia, consideradas especialidades médicas pelo CFM, são as práticas mais lembradas, sendo a acupuntura a mais aceita delas $3,4,15,23$.
As práticas corporais também são relativamente populares em outros estudos, a depender, principalmente, do município ou da região investigados. Fitoterápicos, por sua vez, são mais bem aceitos por outros profissionais da área de saúde, não sendo muito populares na prática médica. Em estudo que avaliou o uso de PIC na população brasileira, a partir dos dados da Pesquisa Nacional de Saúde, porém, a fitoterapia foi a prática mais frequente, embora com baixa prevalência (2,5\% IC95\% 2,2-2,8), seguida da acupuntura $(0,6 \%)$ e da homeopatia $(0,6 \%)^{\mathbf{2 4}}$.

O estudo teve sua abrangência limitada por ser restrito a um município de médio porte, do interior do estado, e, portanto, com um número menor de profissionais vinculados ao SUS, além da pouca oferta de PIC para comparação com outras pesquisas realizadas no País, a maioria em capitais.

\section{Considerações finais}

Em geral, o conhecimento das práticas entre os médicos atuantes no município estudado foi comparável às demais pesquisas, sendo moderado para as práticas já reconhecidas pelo CFM - principalmente para a acupuntura - e pequeno para homeopatia, práticas corporais, massagem. Foi possível verificar que o uso próprio ou de familiares influencia no conhecimento e, com isso, na recomendação de uso aos pacientes, embora nem sempre a recomendação seja realizada formalmente na forma de uma prescrição médica.

Como medidas locais, sugere-se melhorar a divulgação de ofertas em nível municipal e a própria oferta destas nos locais de trabalho. Em relação a melhorar as perspectivas das práticas integrativas dentro do território nacional, pontua-se que a medida de ampliar a disponibilidade das PIC é apenas satisfatória. A ampliação na formação acadêmica durante a graduação - assinalando alternativas terapêuticas, principalmente em afecções crônicas - é 
fundamental para o avanço adequado das PIC, tanto no que tange ao conhecimento quanto na aceitação e recomendação/prescrição delas.

\section{Colaboradores}

Kracik MLA (0000-0003-1518-6007)* participou na concepção e delineamento do estudo, coleta e organização dos dados, análise e interpretação destes, elaborou a primeira versão do manuscrito e aprovou sua versão final. Pereira PMB (0000-0002-9197-8623)* contribuiu para a interpretação dos dados, elaboração e revisão crítica do artigo e aprovação da sua versão final. Iser BPM (0000-00016061-2541)* contribuiu para a concepção e delineamento do estudo, análise e interpretação dos dados, revisão crítica do artigo e aprovação da sua versão final.

\section{Referências}

1. World Health Organization. WHO traditional medicine strategy: 2014-2023. Geneva: WHO; 2013.

2. Monteiro MMS. Práticas integrativas e complementares no Brasil - revisão sistemática [monografia] [internet]. Recife: Fundação Osvaldo Cruz; 2012. 36 p. [acesso em 2015 mar 10]. Disponível em: https:// www.arca.fiocruz.br/handle/icict/28539.

3. Otani PAM, Barros FN. A Medicina Integrativa e a construção de um novo modelo na saúde. Ciênc. Saúde Colet. 2011;16(3):1801-11.

4. Sousa CMI, Hortale AV, Bodstein RCA. Medicina Tradicional Complementar e Integrativa: desafios para construir um modelo de avaliação do cuidado. Ciênc. Saúde Colet. 2018; 23(10):3403-12.

5. Brasil. Ministério da Saúde. Thematic Glossary: Integrative and Complementary Health's Practices. Brasília, DF: Secretaria de Atenção à Saúde; 2018.
6. Strouss L, Mackley A, Guillen U, et al. Complementary and Alternative Medicine use in women during pregnancy: do their healthcare providers know? BMC Complement Altern Med. 2014; (14):85.

7. Koithan M, Bell IR, Niemeyer K, et al. A complex systems science perspective for whole systems of complementary and alternative medicine research. Forsch Komplementmed 2012; 19(supl1):7-14.

8. Peregoy JA, Clarke TC, Jones LI, et al. Regional Variation in Use of Complementary Health Approaches by U.S. Adults. NCHS Data Brief. 2014; (146):1-8.

9. Miranda ASM, Clara MJ. Terapêuticas não convencionais: Perspectivas dos médicos de medicina geral e familiar [dissertação] [internet]. Covilhã: Faculdade de Ciências da Saúde, Universidade da Beira Interior; 2010. 100 f. [acesso em 2015 abr 9]. Disponível em: https://ubibliorum.ubi.pt/handle/10400.6/809. 
10. Nagai SC, Queiroz MS. Medicina complementar e alternativa na rede básica de serviços de saúde: uma aproximação qualitativa. Ciênc. Saúde Colet. 2011; 16(3):1793-1800.

11. Hunt KJ, Coelho HF, Wider B, et al. Complementary and Alternative Medicine Use in England: Results from a National Survey. Int J Clin Pract. 2010; 64(11):1496-1502.

2. Faqueti A, Tesser CD. Utilização de Medicinas Alternativas e Complementares na atenção primária à saúde de Florianópolis/SC, Brasil: percepção de usuários. Ciênc. Saúde Colet. 2018; 23(8):2621-30.

13. Sousa LA, Barros NF. Integrative and Complementary Practices in the Unified Health System: progresses and challenges. Rev. Latino-Am. Enfermagem, 2018; (26):1-2.

14. Fischer FH, Lewith G, Witt CM, et al. High prevalence but limited evidence in complementary and alternative medicine: guidelines for future research. BMC Complement Altern Med. 2014; (14):46.

15. Saad M, Lima TP. Medicina alternativa, complementar e integrativa: problema, dilema e desafio para o campo da saúde. Eistein: Educ Contin Saúde. [internet] 2010 [acesso 2015 abr 12]; 8(3Pt2):148-50. Disponível em http://apps.einstein.br/revista/arquivos/ PDF/1766-EC_V8_N3_pg148-50.pdf.

16. Neto JAC, Sirimarco MT, Neto JAD, et al. Uso e compreensão da medicina alternativa e complementar pela população de Juiz de Fora. HU Revista. 2010; 36(4):266-76.

17. Thiago SCS, Tesser CD. Percepção de médicos e enfermeiros da Estratégia de Saúde da Família sobre terapias complementares. Rev Saúde Pública. 2011; 45(2):249-57.

18. Long AF. Complementary and Alternative Medicine (CAM) and the Public Health: an Innovative Heal- thcare Practice in Supporting and Sustaining Health and Well-Being. Epidemiol. 2013; 4(1):141-6.

19. Junior ET. Práticas integrativas e complementares em saúde, uma nova eficácia para o SUS. Estud. Av. [internet] 2016 [acesso em 2019 junho 10]; 30(86):99-112. Disponível em: http:// www.scielo.br/scielo.php?script=sci_arttext\&pid $=$ S0103-40142016000100099.

20. Islândia MS, Bodstein RCA, Tesser DC, et al. Práticas integrativas e complementares: oferta e produção de atendimentos no SUS e em municípios selecionados. Cad. Saúde Pública 2012; 28(11):2143-54.

21. Sociedade Brasileira de Medicina de Família e Comunidade. Currículo baseado em competências para Medicina de Família e Comunidade [internet]. Rio de Janeiro: SBMFC; 2015. [acesso em 2019 fev 5]. Disponível em https://www.ufrgs.br/telessauders/documentos/biblioteca_em_saude/006_material_saude_ curriculo_competencias_mfc.pdf.

22. Teixeira RAG, Barros RCR, Teixeira USC, et al. Formação de professores de classe hospitalar em saúde mental como resultante de uma pesquisa-ação existencial. Temas educ. saúde, 2017; (13):317-34.

23. Brasil. Ministério da Saúde. Secretaria de Atenção à Saúde. Departamento de Atenção Básica. Política Nacional de Práticas Integrativas e Complementares no SUS: atitude de ampliação de acesso. 2. ed. Brasília, DF: MS, 2015 .

24. Boing AC, Ribeiro Santiago PH, Tesser CD, et al. Prevalence and associated factors with integrative and complementary practices use in Brazil. Complement Ther Clin Pract. 2019 1;(37):1-5.

Recebido em 19/02/2019

Aprovado em 09/09/2019

Conflito de interesses: inexistente

Suporte financeiro: não houve 